\title{
On the Performance of Cooperative Diversity in Infrastructure-Based Networks with Two Relays
}

\author{
Jun Yeop Jung \\ Yonsei University, Dept. of Electrical and Electronic Engineering, \\ 134 Shinchon-Dong, Seodaemun-Gu, Seoul 120-749, Korea \\ amigos97@yonsei.ac.kr \\ http://mcl.yonsei.ac.kr
}

\begin{abstract}
In this paper, the performance of four cooperative relaying schemes, which are classified by requiring a quantity of feedback information, is evaluated in the high SNR region and is compared with that of MIMO relaying schemes with two antennas. For amplify-and-forward (AF) relay, all schemes except the second hop selection scheme provide the second order diversity gain in high SNR region. For decode-andforward (DF) relay, despite using more channel information, the coherent $\mathrm{BF}$ scheme does not offer the second order diversity. A system level simulation is evaluated to analyze the effects of user distribution in terms of the average capacity. For AF relay, the capacity of the cooperative relaying model is higher than that of the MIMO relaying model as about $0.2 \mathrm{bps} / \mathrm{Hz}$. For DF relay, the MIMO relaying model provides capacity gain about larger than $0.3 \mathrm{bps} / \mathrm{Hz}$ for the coherent beamforming and the Alamouti-based scheme since the effect of array gain at relay for these schemes is more dominant factor to increase the capacity compared with the effect of reducing path loss.
\end{abstract}

\section{Introduction}

Recently there has been increasing interest in the concept of augmenting the infrastructure-based networks with relay in order to provide high data rate and expand service coverage in a cost efficient manner[1,2]. Moreover, the present paper evaluates the performance of cooperative diversity using relay. Based on the ideas of user cooperation diversity [3], Laneman et al. [4] propose cooperative protocols for the three-terminal case, and it is shown that diversity gains can be achieved.

In this paper, the performance of four cooperative relaying schemes, which are classified by requiring a quantity of feedback information, is evaluated in the high SNR region and is compared with the performance of MIMO relaying schemes with two antennas. The first, performance is measured in terms of capacity, outage probability and cooperative diversity in only short-term fading channel environment. Then, the performance is investigated in environment where longterm fading is considered for more realistic assumption. 


\section{Channel and Relay Model}

In our scenario, it is assumed that BS and mobiles can communicate via relay, user mobility is low. We assume that the channel is considered time-invariant over at least one transmission cycle (block fading). BS transmission slot separated from relay transmission slot in order to avoid interference. The discretetime baseband equivalent model of the channel with two relays which use same frequency band is shown in Fig.1. From now on, $n^{\prime}$ is used as symbol index of the first slot, and $n$ is used as symbol index of the second slot. For cooperative diversity, a signal during the first slot received at the relay and the received signal during the second slot at the mobile are

$$
r_{i}\left[n^{\prime}\right]=h_{b r_{i}} s\left[n^{\prime}\right]+z_{b r_{i}}\left[n^{\prime}\right]
$$

and

$$
y[n]=h_{r_{1} m} x_{1}[n]+h_{r_{2} m} x_{2}[n]+z_{m}[n]
$$

for $n, n^{\prime}=1, \ldots, N$.

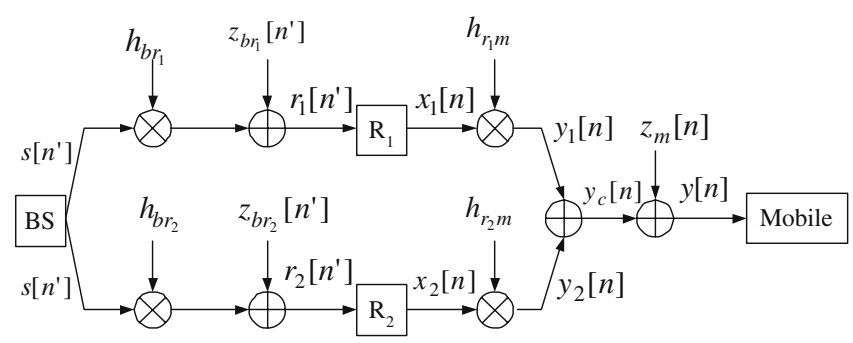

Fig. 1. Discrete-time baseband equivalent relay channel

The BS transmits signal as $s\left[n^{\prime}\right]$ for first slot. During this interval, the $i$-th relay processes received signal, $r_{i}\left[n^{\prime}\right]$ and AF relay retransmits signal

$$
x_{i}[n]=w_{i}[n] g_{i}[n] r_{i}\left[n^{\prime}\right]
$$

for $n, n^{\prime}=1, \ldots, N$. For AF relay case, the received signal at relay is retransmitted after its power was amplified by the following amplifier gain,

$$
g_{i}[n]=\sqrt{\frac{P_{R}}{P_{B}\left|h_{b r_{i}}\right|^{2}+N_{r_{i}}}}
$$

where the amplifier gain depends upon the received power. For DF relay the transmitted signal at relay is re-encoding version of the received signal. We assume that the relay might fully decode without error. The relay transmits the signal

$$
x_{i}[n]=w_{i}[n] \hat{s}\left[n^{\prime}\right]
$$


for $n, n^{\prime}=1, \ldots, N . \hat{s}\left[n^{\prime}\right]$ denotes re-encoded signal of received signal from BS. The total power of two relays transmitted signals is $P_{R}$ for fair comparison among different transmission schemes. $w_{i}[n]$ denotes the weight which depends on transmission schemes. The weight constraint is given by

$$
\left|w_{1}[n]\right|^{2}+\left|w_{2}[n]\right|^{2}=1 \text {. }
$$

\section{Outage Probability Performance}

We focus on nonergodic scenarios, and evaluate performance in terms of outage probability. The channel capacity $C$ for an instantaneous SNR is given by

$$
C=\frac{1}{2} \log \left(1+S N R_{\text {received }}\right)
$$

where $S N R_{\text {received }}$ is the received signal to noise ratio at mobile. For a target spectral efficiency $R, C<R$ denotes the outage event, and $\operatorname{Pr}[C<R]$ denotes the outage probability which can be approximated in high-SNR region.

\subsection{Cooperative Relaying Schemes Using Two Relays with One Antenna}

Four cooperative relaying schemes are considered: the coherent beamforming scheme, Alamouti-based scheme, the optimal selection scheme and the second hop selection scheme. The coherent beamforming scheme uses single antenna of two relays like maximal ratio transmission [8] in MIMO. Alamouti-based scheme is proposed by an Alamouti-based multi-user space-time diversity system of $[6,7]$. Since the scheme in $[6,7]$ uses two frequency bands, modified transmission scheme which use single frequency band is used for fair comparing other schemes. The optimal selection scheme selects the path of better total channel condition out of two relay paths. All CSIs of relay channel should be used for deciding optimal path. Then selected relay transmits signals with power, $P_{R}$ and the other relay stop transmitting. In other words, magnitude of weight of selected relay is one and the other is zero. The second hop selection selects relay which has better channel condition between relay and mobile. It can be regard the sub-optimal scheme, which makes it work with partial information.

For AF relay, the capacity of the coherent beamforming scheme and Alamouti based scheme in table 1 is upper bound, which is the capacity in noiseless at second hop, in order to express the capacity as closed form. To analyze DF relay case, a max-flow min-cut interpretation [5]. Roughly speaking, the rate of the information flow transmitted on the relay channel is constrained by the bottleneck corresponding to either the first cut (BS-relay link) or the second one (relay-mobile). For the coherent beamforming scheme and the Alamouti-based scheme, SNR at mobile can be evaluated easily without using upper bound unlike AF relay case. The process of detailed analysis is omitted and the approximation of outage probabilities for four schemes in high SNR is tabulated in table 1 . 


\subsection{MIMO Relaying Schemes Using One Relay with Two Antennas}

For AF relay transmission, two relays with one antenna scheme has the same performance of the capacity and outage probability as one relay with two antennas scheme when the path loss of geometry is not considered, since each antenna of a relay retransmits only an amplified version of each received signal. For DF relay transmission, the capacity of first hop channel is increased by array gain at receiver. Therefore unlike AF relay case, we can expect that the performance of one relay with two antennas is better than that of two relays with single antennas in terms of capacity outage. Table 1 denotes outage probabilities for 4 schemes. When long-term fading which includes path loss, shadowing is considered, we will show the result by simulation in chapter 4 .

Table 1. Outage Performance

\begin{tabular}{|c|c|c|c|}
\hline Scheme & $\begin{array}{l}2 \text { AF relays with } \\
1 \text { antenna }\end{array}$ & $\begin{array}{l}2 \text { DF relays with } \\
1 \text { antenna }\end{array}$ & $\begin{array}{l}1 \text { DF relay with } \\
2 \text { antennas }\end{array}$ \\
\hline $\begin{array}{c}\text { Coherent } \\
\text { beamforming }\end{array}$ & $\frac{1}{2 \sigma_{b r_{1}}^{2} \sigma_{b r_{2}}^{2}}\left(\frac{2^{2 R}-1}{S N R}\right)^{2}$ & $\left(\frac{1}{\sigma_{b r_{1}}^{2}}+\frac{1}{\sigma_{b r_{2}}^{2}}\right)\left(\frac{2^{2 R}-1}{S N R}\right)$ & $\begin{array}{c}\frac{1}{2}\left(\frac{1}{\sigma_{b r_{1}}^{2} \sigma_{b r_{2}}^{2}}+\frac{1}{\sigma_{r_{1} m}^{2} \sigma_{r_{2} m}^{2}}\right) \\
\cdot\left(\frac{2^{2 R}-1}{S N R}\right)^{2}\end{array}$ \\
\hline $\begin{array}{l}\text { Optimal } \\
\text { selection }\end{array}$ & $\begin{array}{c}\left(\frac{1}{\sigma_{b r_{1}}^{2}}+\frac{1}{\sigma_{r_{1} m}^{2}}\right) \\
\cdot\left(\frac{1}{\sigma_{b r_{2}}^{2}}+\frac{1}{\sigma_{r_{2} m}^{2}}\right) \\
\cdot\left(\frac{2^{2 R}-1}{S N R}\right)^{2}\end{array}$ & $\begin{array}{c}\left(\frac{1}{\sigma_{b r_{1}}^{2}}+\frac{1}{\sigma_{r_{1} m}^{2}}\right) \\
\cdot\left(\frac{1}{\sigma_{b r_{2}}^{2}}+\frac{1}{\sigma_{r_{2} m}^{2}}\right) \\
\cdot\left(\frac{2^{2 R}-1}{S N R}\right)^{2}\end{array}$ & $\begin{array}{c}\left(\frac{1}{2 \sigma_{b r_{1}}^{2} \sigma_{b r_{2}}^{2}}+\frac{1}{\sigma_{r_{1} m}^{2} \sigma_{r_{2} m}^{2}}\right) \\
\cdot\left(\frac{2^{2 R}-1}{S N R}\right)^{2}\end{array}$ \\
\hline $\begin{array}{c}\text { Alamouti- } \\
\text { based }\end{array}$ & $\frac{1}{\sigma_{b r_{1}}^{2} \sigma_{b r_{2}}^{2}}\left(\frac{2^{2 R}-1}{S N R}\right)^{2}$ & $\left(\frac{1}{\sigma_{b r_{1}}^{2}}+\frac{1}{\sigma_{b r_{2}}^{2}}\right)\left(\frac{2^{2 R}-1}{S N R}\right)$ & $\begin{array}{c}\frac{1}{2}\left(\frac{1}{\sigma_{b r_{1}}^{2} \sigma_{b r_{2}}^{2}}+\frac{4}{\sigma_{r_{1} m}^{2} \sigma_{r_{2} m}^{2}}\right) \\
\cdot\left(\frac{2^{2 R}-1}{S N R}\right)^{2}\end{array}$ \\
\hline $\begin{array}{c}\text { Second hop } \\
\text { selection }\end{array}$ & $\begin{array}{c}\left(\frac{\sigma_{b r_{1}}^{-2} \sigma_{r_{2} m}^{-2}+\sigma_{b r_{2}}^{-2} \sigma_{r_{1} m}^{-2}}{\sigma_{r_{1} m}^{-2}+\sigma_{r_{2} m}^{-2}}\right) \\
\cdot\left(\frac{2^{2 R}-1}{S N R}\right)\end{array}$ & $\begin{array}{c}\left(\frac{\sigma_{b r_{1}}^{-2} \sigma_{r_{2} m}^{-2}+\sigma_{b r_{2}}^{-2} \sigma_{r_{1} m}^{-2}}{\sigma_{r_{1} m}^{-2}+\sigma_{r_{2} m}^{-2}}\right) \\
\cdot\left(\frac{2^{2 R}-1}{S N R}\right)\end{array}$ & $\begin{array}{c}\left(\frac{1}{2 \sigma_{b r_{1}}^{2} \sigma_{b r_{2}}^{2}}+\frac{1}{\sigma_{r_{1} m}^{2} \sigma_{r_{2} m}^{2}}\right) \\
\cdot\left(\frac{2^{2 R}-1}{S N R}\right)^{2} \\
\end{array}$ \\
\hline
\end{tabular}

\section{Simulation Results}

Fig. 2 shows the outage probability performance of AF and DF relay case in symmetric networks in which the fading variances are identical, e.g., $\sigma_{b r_{i}}^{-2}=$ $\sigma_{r_{i} m}^{-2}=1$. For AF relay, all schemes except the second hop selection scheme provide the second order diversity gain in high SNR. Even though the Alamoutibased scheme does not require feedback information, the second diversity is achieved. For DF relay, despite using more channel information, the coherent BF scheme does not offer the second order diversity. Since the relay received signal should be fully decoded at the relay, the relay channel capacity is limited by the capacity of first hop (BS-relay link). The coherent beamforming scheme has rather normalized SNR loss of $3 \mathrm{~dB}$ than the second hop selection schemes which require only relay-mobile channel information in high SNR region. 


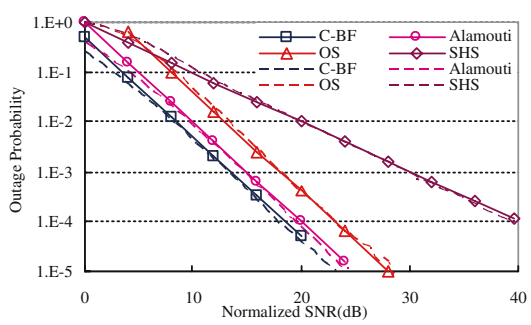

(a) AF relay case

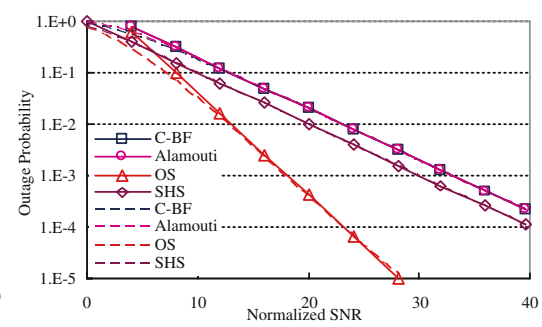

(b) DF relay case

Fig. 2. Outage probabilities of cooperative transmission

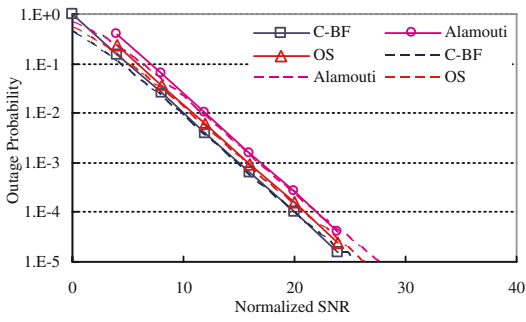

(a)

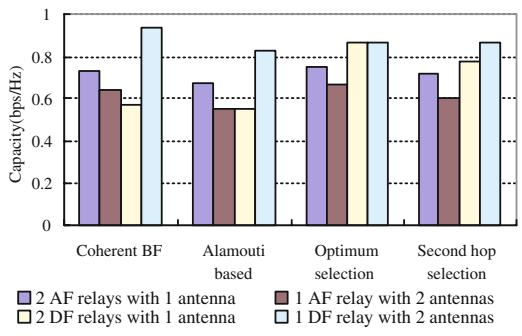

(b)

Fig. 3. (a) Outage probabilities of MIMO relay transmission (b) Average capacity

For AF relay transmission, the performance is identical between two relays with a single antenna and one relay with two antennas if we do not consider the path loss by effects due to the geometry. Fig. 3 (a) shows the outage probability performance of DF relay with two antennas in symmetric networks. In contrast to two relay with one antenna case, the coherent BF scheme and the Alamouti based scheme can offer the second order diversity since the capacity of BS-relay channel is increased by the array gain of relay antennas.

Fig. 3 (b) shows the average capacity which is obtained by system level simulation. We follow the evaluation methodology [9] submitted to 3GPP2 (3rd Generation Partnership Project 2) specification for the evaluation of cdma2000. The cell is divided by three sectors and only one sector is considered. Rayleigh fading channel based on Jake's model is considered and all channel elements are assumed to be independent. Mobile speed is fixed to $3 \mathrm{~km} / \mathrm{h}$.

\section{Concluding Remarks}

For AF relay, all schemes except the second hop selection scheme provide the second order diversity gain in high SNR region. Even though the Alamoutibased scheme does not require feedback information, the second diversity is achieved. For DF relay, despite using more channel information, the coherent BF 
scheme does not offer the second order diversity. Since the relay received signal should be fully decoded at the relay, the relay channel capacity is limited by the capacity of first hop (BS-relay link). The coherent beamforming scheme has rather normalized SNR loss of $3 \mathrm{~dB}$ than the second hop selection schemes which require only relay-mobile channel information in high SNR region. A system level simulation is evaluated to analyze the effects of user distribution in terms of the average capacity. For AF relay, the capacity of the cooperative relaying model is higher than that of the MIMO relaying model as about $0.2 \mathrm{bps} / \mathrm{Hz}$ since AF relay can reduce the effect of path loss. For DF relay, the MIMO relaying model provides remarkable capacity gain about larger than $0.3 \mathrm{bps} / \mathrm{Hz}$ for the coherent beamforming and the Alamouti-based schemes since the effect of array gain at relay for these schemes is more dominant factor to increase the capacity compared with the effect of reducing path loss. For the selection combining-based schemes such the optimum selection scheme and the second selection scheme, the capacity of the cooperative relaying model and the MIMO relaying model are similar, since the effect of array gain is similar to that of reducing path loss.

\section{References}

1. H. Yanikomeroglu "Fixed and mobile relaying technologies for cellular networks," Second Workshop on Applications and Services in Wireless Networks (ASWN'02), pp. 75-81, 3-5 July 2002, Paris, France.

2. R. Bruno and M. Conti, "Mesh networks: commodity multihop ad hoc networks", IEEE Communications Magazine, vol. 43, vol. 3, pp. 123-131, March 2005.

3. J. Nicholas Laneman, David N. C. Tse, and Gregory W. Wornell "Energy-efficient antenna sharing and relaying for wireless networks," in Proc.of IEEE Wireless Commun. and Networking Conf., Chicago, IL, March 2000, vol. 1, pp. 7 - 12.

4. J. Nicholas Laneman, David N. C. Tse, and Gregory W. Wornell "Cooperative Diversity in Wireless Networks: Efficient Protocols and Outage Behavior," IEEE Trans. Inform. Theory, vol. 50, no. 12, pp. 3062-3080, Dec. 2004.

5. T. M. Cover and J. A. Thomas, Elements of Information Theory. New York:Wiley, 1991.

6. P.A. Anghel, M. Kaveh, "On the performance of distributed space-time coding systems with one and two non-regenerative relays," IEEE Transactions on Wireless Communications, to appear in 2005.

7. P. A. Anghel, G. Leus, M. Kaveh, "Multi-user space-time coding in cooperative networks," in Proc. of the International Conference on Acoustics, Speech, and Signal Processing (ICASSP'03), Hong Kong, April 6-10, 2003, vol. 4, pp. 73-6.

8. T. Lo. Maximal ratio transmission. IEEE Trans. Comm., 47(10), 1458-1461, October 1999.

9. 3GPP2 C.R1002-0 Version 1.0, "1xEV-DV Evaluation Methodology, Revision 0," December 10, 2004. 